Service social

\title{
Les inondations de juillet 1996 : une série d’événements stressants
}

\section{Suzie Robichaud, Danielle Maltais, Gilles Lalande, Anne Simard et Guy Moffat}

Volume 48, numéro 1, 2001

URI : https://id.erudit.org/iderudit/006875ar

DOI : https://doi.org/10.7202/006875ar

Aller au sommaire du numéro

Éditeur(s)

École de service social de l'Université Laval

ISSN

0037-2633 (imprimé)

1708-1734 (numérique)

Découvrir la revue

Citer cet article

Robichaud, S., Maltais, D., Lalande, G., Simard, A. \& Moffat, G. (2001). Les inondations de juillet 1996 : une série d'événements stressants. Service social, 48(1), 16-33. https://doi.org/10.7202/006875ar
Résumé de l'article

Une catastrophe naturelle ne correspond pas seulement à l'arrivée d'un malheur effroyable et subit, mais constitue un long processus dans lequel s'engagent les sinistrés. Dans la logique de cette perspective, par la présente réflexion, nous nous proposons d'examiner, à l'aide des écrits scientifiques et des entrevues menées auprès de victimes des inondations survenues dans une région du Québec - le Saguenay - en juillet 1996, les trois points qui vont suivre. Tout d'abord, il y a lieu de voir comment les chercheurs analysent les difficultés qui surviennent à la suite d'un désastre. Il importe ensuite de s'attarder aux considérations méthodologiques, aux caractéristiques sociodémographiques et à l'état de santé postdésastre des sinistrés. Et, enfin, il s'agit d'analyser les principales situations stressantes qui se sont imposées aux sinistrés tout au long du parcours. Il sera alors possible pour les intervenants sociaux de mieux comprendre et aider les victimes, et ce, tout au long du processus. 


\title{
Les inondations de juillet 1996 : une série d'événements stressants
}

\author{
Suzie ROBICHAUD \\ Danielle MALTAIS \\ Gilles LALANDE \\ Anne SIMARD \\ Guy MOFFAT
}

Une catastrophe naturelle ne correspond pas seulement à l'arrivée d'un malheur effroyable et subit, mais constitue un long processus dans lequel s'engagent les sinistrés. Dans la logique de cette perspective, par la présente réflexion, nous nous proposons d'examiner, à l'aide des écrits scientifiques et des entrevues menées auprès de victimes des inondations survenues dans une région du Québec - le Saguenay - en juillet 1996, les trois points qui vont suivre. Tout d'abord, il y a lieu de voir comment les chercheurs analysent les difficultés qui surviennent à la suite d'un désastre. II importe ensuite de s'attarder aux considérations méthodologiques, aux caractéristiques sociodémographiques et à l'état de santé postdésastre des sinistrés. Et, enfin, il s'agit d'analyser les principales situations stressantes qui se sont imposées aux sinistrés tout au long du parcours. II sera alors possible pour les intervenants sociaux de mieux comprendre et aider les victimes, et ce, tout au long du processus.

A natural disaster not only involves the appearance of a sudden and appalling calamity but also constitutes a long ongoing process in which the victims are totally involved, a series of events filled with pitfalls in which ideas, emotions, and feelings clash. By using this perspective to analyze scientific evidence and interviews with the victims of the floods of July 1996 in the Saguenay region of Québec, this paper proposes to examine the three following points. First of all, it is important to understand how researchers analyze the difficulties that arise following a disaster. Secondly, it is essential to see these methodological considerations. Finally, there remains the analysis of the principal stressful situations imposed on victims throughout the event. 
Pour l'individu comme pour la collectivité, rien n'est stable. Les destins des hommes comme ceux des villes sont balayés. ... II faut donc penser à tout et affermir son âme contre tout ce qui peut arriver.

Sénèque

À l'aube de ce nouveau millénaire, les individus et les collectivités sont de plus en plus menacés par les désastres naturels (Dufour, 1997; Hare, 1996; Pickering et Owen, 1994). La région du Saguenay - au Québec - n'y échappe pas, comme en fait foi la plus importante perturbation à y survenir au cours des deux dernières décennies. En effet, l'impact des pluies diluviennes des 19, 20 et 21 juillet 1996, associé à la présence d'un réseau hydraulique d'envergure, a provoqué des inondations majeures qui ont touché 39 municipalités et plus de 16000 personnes. Faut-il rappeler l'aspect le plus sombre de cette tragédie? Deux enfants y ont trouvé la mort. Le climat de terreur et la désolation associée aux pertes matérielles, financières, sentimentales et sociales affectent les individus dans leur quotidienneté, puis entraînent une série de conséquences qui peuvent s'échelonner sur une longue période de temps. Pour Murphy (1986), la période que l'on qualifie de postdésastre peut durer de un à dix ans, alors que Green et Lindy (1994) la prolongent à dix-sept ans, tout en soulignant que les séquelles peuvent perdurer pendant toute la vie d'une personne.

Quoi qu'il en soit, un désastre ne correspond pas seulement à l'arrivée d'un malheur effroyable et subit, mais constitue un long processus dans lequel s'engagent les sinistrés, une marche pleine d'embûches. En parcourant ce difficile trajet, peut-être réussironsnous à saisir l'ensemble des effets qu'un désastre peut produire chez les victimes. Oui, il y a déluge sur la ville. Mais l'eau dans les yeux des sinistrés est celle qu'on voit le moins, dira Carol Lebel (1997).

Pour la clarté du propos, il convient avant tout de présenter en trois temps le cadrage de cet article. Tout d'abord, il y a lieu de dégager, à travers les écrits scientifiques, les principales compréhensions et significations que les chercheurs attribuent à un 
désastre, puis de souligner les précisions que certains d'entre eux apportent pour décrire l'expérience des victimes. Ensuite, il est important de voir les considérations méthodologiques, les caractéristiques sociodémographiques et l'état de santé postdésastre des répondants. Enfin, à la lumière des interviews, il s'agit d'examiner les différentes étapes par lesquelles passent les sinistrés. Ainsi, la prise en compte des principales difficultés que vivent les personnes qui ont perdu leur maison permettra aux intervenants sociaux de mieux comprendre et aider les victimes, et ce, tout au long du processus.

\section{DÉLIMITATION CONCEPTUELLE}

Les désastres, qu'ils aient des causes naturelles ou des causes technologiques ou humaines, peuvent être étudiés selon une pluralité de points de vue. Certains auteurs les abordent avec une conception globale et holistique (Barton, 1969; Turner, 1976), tandis que d'autres ont recours à des perspectives plus psychologiques ou sociologiques (Lechat, 1979; Kingston et Rosser, 1974; Logue et collab., 1981).

Ainsi, pour Barton (1969), un désastre correspond à une altération brutale ou à une rupture des conditions de vie auxquelles s'attendent les membres d'un système social. Cette rupture provoque une importante situation de stress collectif. Pour Turner (1976), un désastre constitue un événement menaçant, pour une société ou pour une de ses sous-divisions autosuffisantes, qui occasionne des conséquences indésirables découlant d'une interruption de l'ordre social habituel ou d'un manque de moyens pour l'assurer. De plus, cette situation est culturellement jugée inacceptable par la population. D'autres chercheurs dépeignent les désastres comme des circonstances de stress collectif dont on peut analyser les répercussions sur les individus en considérant et en analysant les éléments qui bouleversent le système social en entier (Kingston et Rosser, 1974). Ainsi, face aux perturbations excessives, la capacité d'ajustement ou d'adaptation des communautés se voit grandement altérée (Lechat, 1979).

Les désastres peuvent être perçus comme des épisodes fragmentés, de par les différentes phases ou étapes qui les caractérisent (Tyhurst, 1951; Powell et Rayner, 1952; Raphael, 
1975; Golec, 1983). La durée de chacune de ces étapes est variable et celles-ci s'acheminent, tant pour les individus que pour les communautés affectés, vers un rétablissement global ou partiel. Sur le plan individuel, Ollendick et Hoffman (1982) se réfèrent au National Institute of Mental Health (NIMH) pour décrire les phases traversées par les victimes. La première constitue la phase "héroïque », qui se manifeste généralement dans la première semaine suivant le désastre; la deuxième, nommée " lune de miel », débute habituellement dans la semaine qui suit et peut s'étendre jusqu'à six mois; y succède la phase de "désillusion », dont la durée peut varier de six mois à deux ans. La phase de "reconstruction », enfin, est entreprise après un ou deux ans.

Toutefois, lorsqu'il est question d'étudier le concept lui-même, plus précisément de définir un désastre, plusieurs auteurs préfèrent s'en tenir à l'utilisation de l'expression " événement traumatisant » (Solomon et collab., 1987; Murphy, 1986). Pour l'Association américaine de psychiatrie (American Psychiatric Association, 1996), "l'événement traumatisant" se situe à l'extérieur des expériences humaines inhabituelles et peut être visiblement affligeant pour n'importe qui. Selon cette approche, certaines expériences sont qualifiées a priori de traumatisantes, comme les menaces à la vie, les désastres naturels et technologiques.

Pour sa part, Murphy (1986) associe les désastres à une série de situations parmi les plus traumatisantes que peuvent connaître les individus. L'auteur parle d'une série d'événements catastrophiques, bien qu'ils soient souvent vus et étudiés comme des épisodes uniques. Notamment, Murphy estime que la catastrophe n'est pas un fait isolé, mais une circonstance qui s'insère dans un continuum d'événements. Dans le même ordre d'idées, Rangell (1976) parle de l'aspect « cumulatif » du traumatisme à la suite de la prise de conscience de la destruction. Berren (1980) a lui aussi utilisé le terme événements (et non événement) pour définir les désastres de tous types.

Cela dit, d'autres précisions sont apportées par des auteurs. En effet, certains soulignent, entre autres choses, que l'évacuation et les nombreux déplacements constituent des étapes difficiles (Gleser et collab., 1981). Le déplacement géographique, somme 
toute, correspond à un stress psychologique important (Fullilove, 1996). D'autres chercheurs ajoutent que la perte de biens personnels et les difficultés économiques rencontrées ne font qu'alourdir le processus de relocalisation (Steinglass et Gerrity, 1990). Dans de telles conditions, l'adaptation à un nouvel environnement ne se fait pas sans peine: sinistrés, famille et couple se trouvent alors déstabilisés (Ursano et McCarroll, 1994; Raphael, 1986). Car perdre sa demeure, c'est aussi perdre une partie de son identité (Steinfield, 1982) ou de sa sécurité (Rowles, 1983). En effet, les individus ont l'impression d'avoir été trompés, puisque la sécurité sur laquelle ils comptaient n'existait pas vraiment (Crabbs et Heffron, 1981). De plus, les crises qui s'ensuivent sont alimentées par différents facteurs: personnels, sociaux, politiques et environnementaux (Belter et Shannon, 1993; Murphy, 1986; Bolin, 1985). Par exemple, le fait d'avoir investi beaucoup dans leur demeure influe sur la manière dont les personnes seront psychologiquement affectées. Quoi qu'il en soit, considérer les désastres comme une série ou comme un cumul de circonstances perturbatrices ou déstabilisantes est essentiel aux fins de notre propos qui tente de montrer comment les inondations qu'a connues la population du Saguenay à l'été 1996 s'inscrivent dans une continuité de faits.

\section{MÉTHOdOLOGIE}

L'observation dégagée dans ces pages n'est pas simplement un questionnement sur le phénomène étudié, mais elle se fonde sur une enquête (Maltais, Robichaud, Simard, 1999). La recherche qualitative $^{1}$ dont il est question ici a été effectuée dans la région du Saguenay. Elle avait pour but de documenter les itinéraires résidentiels des sinistrés, d'analyser les conséquences des inondations de juillet 1996 sur les populations touchées et de connaître les événements auxquels les sinistrés ont dû faire face. L'étude a été effectuée auprès de 69 individus qui ont perdu leur maison et tous leurs biens dans les inondations. Les sinistrés qui

Nombreux sont les chercheurs qui mettent en évidence l'utilité des approches qualitatives lorsque la démarche s'inscrit dans une perspective de compréhension des expériences de la vie quotidienne et lorsqu'elle cherche à comprendre le sens que donnent les individus à un événement (Deslauriers, 1991; Lincoln et Guba, 1985, etc.). 
ont participé à l'enquête - tous des propriétaires occupant leur demeure - ont été choisis au hasard à partir de la liste des victimes des inondations de deux municipalités durement touchées par le désastre. Mentionnons toutefois qu'un nombre restreint d'individus (cinq) ont été rencontrés à la suite de suggestions faites par des connaissances ou par d'autres sinistrés. Les entrevues, qui visaient à recueillir le plus largement possible le point de vue des acteurs interrogés, se sont déroulées entre les mois de janvier et d'avril 1998, soit deux ans après les inondations de juillet 1996, et ont duré en moyenne 2 heures 30 minutes. Seulement huit sinistrés contactés ont refusé de participer à la recherche, disant être encore trop affectés par l'événement. Le taux de réponse s'élève donc à $89,6 \%$. Les entrevues individuelles ont été transcrites intégralement et introduites dans le logiciel Nud-Ist. Une analyse thématique a été réalisée pour dégager les principaux événements qui ont été vécus par les sinistrés.

Soulignons également que, pour relier l'observation à l'explication, deux instruments de collecte de données ont été utilisés. Le premier, le guide d'entrevue, proposait 31 questions ouvertes et un ensemble de thèmes secondaires qui laissaient la possibilité aux sinistrés de s'exprimer sur différents thèmes (pertes subies, itinéraires résidentiels, embûches et problèmes rencontrés, étapes à franchir, etc.). Quant au second outil, il permettait de recueillir des informations sur les caractéristiques sociodémographiques.

\section{CARACTÉRISTIQUES SOCIODÉMOGRAPHIQUES ET ÉTAT DE SANTÉ POSTDÉSASTRE DES RÉPONDANTS ${ }^{2}$}

II y a presque autant d'hommes (50,7 \%) que de femmes $(49,3 \%)$ qui ont accepté de rencontrer les intervieweurs. La majorité des sujets $(53,8 \%)$ avaient moins de 50 ans et étaient mariés ou conjoints de fait $(83,6 \%)$. La plupart des individus $(58,2 \%)$ avaient un niveau de scolarité inférieur ou égal à une $5^{\mathrm{e}}$ secondaire et leur revenu annuel brut, en 1997, était inférieur à $35000 \$(76,2 \%)$. En ce qui a trait à l'état de santé postdésastre,

2 Certaines des idées émises dans les parties qui vont suivre se retrouvent dans l'article Redéfinition de l'habitat et santé mentale des sinistrés suite à une inondation (Maltais, Robichaud et Simard, 2000). 
près d'un sujet sur deux (42\%) estime que son état de santé s'est détérioré depuis les inondations de juillet 1996, tandis qu'un peu plus du tiers des répondants $(36,4 \%)$ mentionnent l'apparition de nouveaux problèmes de santé, de malaises et de douleurs. Un sujet sur dix $(11 \%)$ déclare avoir abandonné volontairement ses activités professionnelles, parce que la pression était trop élevée, et avoir subi des interruptions involontaires de travail à la suite des inondations (11\%).

\section{UN DÉSASTRE : UNE SUITE D'ÉVÉNEMENTS}

Les entrevues réalisées auprès des victimes des inondations de 1996 autorisent à regarder de plus près les différents décors qui composent leur paysage, et ce, tout au long du trajet. Ceux-ci s'insèrent dans onze événements qui ont pu être clairement identifiés et qui se succèdent dans le temps. Chacun d'eux intègre les différents obstacles que traversent les victimes et rend possible le constat suivant: un désastre ne constitue pas un fait isolé, mais représente une suite d'événements; ce que plusieurs auteurs ont observé (Belter et Shannon, 1993; Murphy, 1986; Rangell, 1976, etc.), mais qu'aucun n'a présenté de façon détaillée. Regardons à présent, de plus près, les diverses situations auxquelles les sinistrés ont été confrontés.

\section{a) La menace}

La plupart des sinistrés se trouvaient à leur domicile au moment des inondations de juillet 1996 (82,5\%). II semble toutefois que le danger perçu était moins grand que le danger réel, puisque les gens avaient tendance à sous-estimer l'ampleur de la catastrophe et à nier la gravité de la situation. En fait, la situation et le comportement des cours d'eau étaient associés aux crues printanières. Toutefois, l'inquiétude s'est accrue graduellement. Certaines personnes se sont également dites très effrayées lorsqu'elles ont senti bouger la maison et entendu trembler les vitres des fenêtres. D'autres, sous l'impulsion du moment, ont mis leur vie en danger en tentant de récupérer certains objets. Avec la montée graduelle des eaux, la peur s'est installée. Alors, la plupart des femmes ayant la garde des enfants se sont préoccupées de la sécurité de ces derniers. Ce sont elles qui ont réclamé le départ 
du domicile lorsque l'évacuation ne faisait pas l'objet d'une demande explicite des autorités de la sécurité civile.

\section{b) L'évacuation}

Les pluies se sont intensifiées. La majorité des sinistrés ont quitté leur domicile. Certains ont résisté jusqu'au moment où l'eau s'est infiltrée dans la demeure. Sortir de chez soi, sans pouvoir emporter quoi que ce soit, sinon quelques vêtements, un peu d'argent, quelques souvenirs, certains papiers importants (passeports, certificats de naissance et actes de mariage, photographies, etc.) ou rien, parce que l'on agit ou réagit de façon automatique, constitue une catastrophe en soi. Dans l'affolement, la presque totalité des sinistrés $(92 \%)$ se sont rendus instinctivement dans des endroits familiers, soit chez des membres de leur famille immédiate ou chez des amis avec qui ils entretenaient des relations privilégiées. Les centres d'hébergement collectif mis à la disposition de la population évacuée ont également été utilisés par quelques répondants ( $8 \%$ ). Ceux-ci n'y sont toutefois demeurés que quelques heures ou quelques jours, préférant aller vivre dans un autre hébergement temporaire où ils ont résidé pendant quelques mois.

\section{c) L'attente d'information}

L'accès aux sites inondés est interdit. Les sinistrés se trouvent donc plongés dans l'incertitude. C'est alors l'attente d'information, d'une autorisation qui lèvera l'interdiction de se rendre sur les lieux du sinistre et permettra, somme toute, de constater l'état de sa résidence et de ses biens. Cette période de doute est décrite comme particulièrement stressante et éprouvante. Les individus se sentent dépossédés, appauvris, et l'épuisement commence à se faire sentir. Les sinistrés se posent alors les questions suivantes : "Qu'adviendra-t-il de nous? Comment s'organiseront les secours? Recevrons-nous des compensations en cas de pertes importantes? ॥

\section{d) L'instant de vérité}

Tant que les sinistrés n'ont pas vu les dégâts, ils ne réalisent pas l'ampleur du désastre. Ainsi, le retour à sa demeure, à tout le 
moins à ce qui en reste, si reste il y a, parce qu'elle peut avoir été emportée par la rivière, permet de mesurer le sérieux de la situation et la gravité des dommages. Ce moment de vérité provoque de fortes réactions émotives. En effet, la perte de sa maison et des objets les plus significatifs est un autre choc qui déclenche la désolation, le découragement, la tristesse. La colère quant à elle reste secrète pour certains, alors que chez d'autres elle explose : on pleure, on crie, on frappe. Des personnes en général peu démonstratives craquent et s'effondrent. Et d'autres questions surgissent. En effet, les sinistrés se demandent : "Que va-t-il nous arriver? Quoi faire pour sortir de cette crise? Va-t-on nous aider? " La plupart de ces préoccupations restent présentes jusqu'à ce que se mettent en place les services d'aide aux victimes.

\section{e) Un itinéraire résidentiel}

Après les inondations, les sinistrés ont vécu en moyenne dans trois milieux de vie différents. Le premier endroit de relogement a été choisi sous le signe de l'urgence et visait à combler les besoins de s'abriter, de dormir, de manger et de retrouver un peu de calme et de sécurité. Mais, lorsque les sinistrés ont pris conscience qu'ils avaient tout perdu et qu'ils ne pouvaient retourner dans leur ancien domicile, ils ont alors désiré s'installer dans des endroits suffisamment grands pour pouvoir reprendre le rythme normal de la vie quotidienne. On souhaitait recréer un second chez-soi et laisser ainsi libre cours aux pleurs et à la manifestation d'inquiétudes, voire d'incertitudes. La location d'un appartement ou d'une maison ainsi que l'utilisation d'installations récréatives comme des chalets ou des roulottes ont été privilégiées par la plupart des sinistrés (72\%).

Toutefois, malgré la bonne volonté des répondants de recréer un environnement où il était agréable de vivre, tous ont mentionné s'être sentis à l'étroit et vivre dans des environnements totalement étrangers. L'absence de biens et d'objets familiers, la perte de points de repère, la proximité de voisins bruyants et turbulents ainsi que la petitesse des terrains sont les principales raisons évoquées par les sinistrés pour expliquer qu'il a été difficile de se sentir chez soi dans les différents types de ressources utilisés. Bref, l'itinéraire résidentiel des répondants a été ponctué 
d'incertitudes, de tracas et de difficultés de toutes sortes, diminuant leur capital d'énergie.

\section{f) Une foule de démarches}

Toutes les personnes sinistrées ont vécu péniblement l'ensemble des démarches qu'elles ont dû effectuer auprès de différentes instances: gouvernementales, municipales ou communautaires. Le recours inhabituel à ces différentes formes d'aide provoque une certaine agitation, soulève tantôt la colère, tantôt la gêne, parfois même une certaine humiliation. II faut faire la file, attendre des heures afin de recevoir des bons d'achat qui permettront de se procurer l'essentiel. Les emplettes, souvent effectuées à la hâte, causent une certaine insatisfaction. Toutes ces opérations obligent les individus à discuter, argumenter, négocier avec des personnes qui montrent parfois peu d'empathie. Cette insensibilité se manifeste chez des représentants d'organismes, mais également chez certains membres de la famille. Outrés par l'infantilisme des procédures (obligation de recourir à un notaire, chèque émis au nom de la banque, etc.), les sinistrés ont des sursauts de révolte. Tout au moins, ces diverses sollicitations entraînent de nombreuses frustrations, de la déception, de l'amertume encore bien perceptibles. En effet, le souvenir de ces contraintes laisse un goût amer.

\section{g) L'attente des réponses}

La période d'attente concernant les différentes demandes d'aide formulées entraîne des réactions d'inquiétude et déclenche une insécurité financière. Aux appréhensions vient s'ajouter l'irritation face aux délais que l'on doit subir. Par exemple, un couple doit attendre 65 jours avant de connaître le montant qui lui sera versé à titre compensatoire. Les victimes se sentent donc agitées, désarmées et souvent impuissantes. Néanmoins, les sinistrés doivent continuer à faire valoir leurs droits. À cette obligation s'ajoutent d'autres frustrations comme celle qui résulte d'une demande de révision lorsque la somme compensatoire versée est bien au-dessous de la valeur réelle des biens. 


\section{h) Le relogement}

Forcés de reconstruire leur domicile, plusieurs sinistrés évoquent une certaine anesthésie émotive. La priorité est accordée aux démarches associées aux travaux de reconstruction (démolition, nettoyage, aménagement du terrain, etc.) et à d'autres impératifs (permis de démolition, arpentage, permis de construction, etc.). Chacune de ces tâches devient une source de préoccupation, de tension qui débouche sur des conflits familiaux.

Si certains sinistrés ont la possibilité de rebâtir leur demeure au même endroit, d'autres, par contre, choisissent d'en changer l'emplacement ou se voient dans l'obligation de le faire. Engagés dans le processus de deuil à la suite de la disparition de leur maison, les sinistrés doivent faire face au paradoxe suivant : aller de l'avant malgré les incertitudes et les obligations d'assumer le quotidien dans un contexte où toutes les bases servant à assurer l'équilibre sont anéanties ou profondément bouleversées (environnement physique, social, familial, culturel et économique). Sur le plan environnemental, par exemple, la modification s'observe comme suit. Lors des inondations, la plupart des répondants possédaient des terrains dont la dimension était supérieure à 6000 pieds carrés $(92,5 \%)$ et six sujets sur dix $(60,0 \%)$ avaient accès à des terrains dont la superficie était supérieure à 21000 pieds carrés. Des répondants encore propriétaires, seulement $28,5 \%$ possèdent à présent un terrain dont la superficie est supérieure à 21000 pieds carrés. Bref, près d'un sujet sur deux $(43,0 \%)$ dispose maintenant d'un terrain de dimensions inférieures. Quoi qu'il en soit, les changements, qui supposent un ajustement sur le plan de l'organisation personnelle et des relations avec les autres, apportent de nombreuses contraintes: l'éloignement de la famille, la perte de certains amis, l'aménagement dans une habitation non conforme à ses attentes ou à ses besoins.

\section{i) Les emprunts bancaires}

La majorité des victimes ont dû effectuer des emprunts pour faire face aux pertes subies : meubles, biens personnels, équipements sportifs et informatiques, automobile, bateau, etc. C'est toutefois le prêt hypothécaire qui constitue la plus grande dette contractée. 
En effet, $62,9 \%$ des répondants n'avaient aucun emprunt hypothécaire avant les inondations, tandis qu'à l'heure actuelle seulement $14,3 \%$ se trouvent dans la même situation. La moyenne du prêt hypothécaire est plus élevée de 13000 \$ pour les sinistrés possédant déjà un prêt hypothécaire, tandis qu'elle se situe à $38692 \$$ pour ceux qui n'avaient pas d'hypothèque sur la maison détruite par les eaux. Cet endettement provoque déceptions, irritations et angoisses. Mentionnons que, dans l'ensemble, les sinistrés estiment n'avoir reçu, par la voie de l'aide financière gouvernementale ou charitable, qu'environ la moitié de la valeur réelle de ce qu'ils ont perdu. D'ailleurs, il ressort que le montant des pertes, estimé à six millions de dollars, n'inclut pas l'endettement généré par les nouveaux prêts hypothécaires ni la suppression des revenus générés par les emplois exercés dans l'ancien domicile (gîte du passant, édition, garderie en milieu familial, couture, etc.).

\section{j) La gestion des malaises et des deuils}

Une fois que les tâches associées à la reconstruction ou au relogement sont terminées, les gens prennent conscience, une fois de plus, de l'ensemble des pertes qui sont les leurs. De plus, ils éprouvent divers malaises que le changement de domicile ou de quartier apporte dans leur vie. Les dettes constituent ainsi une source importante d'anxiété et de préoccupation, puis apportent un lot de déceptions. II faut faire le deuil d'une sécurité établie, modifier ses projets d'avenir, par exemple en repoussant le moment de la retraite. Le manque d'énergie, d'entrain et d'enthousiasme face à la vie ainsi que l'hébergement ont fait que plusieurs individus ont diminué la fréquence des échanges sociaux $(25,4 \%)$ ainsi que leurs activités de loisir $(18 \%)$. Les tensions avec la famille élargie $(19,4 \%)$ tout comme les malentendus entre conjoints ne sont pas rares (15\%). Qui plus est, certains sinistrés rapportent avoir connu des changements importants dans leur vie professionnelle: diminution de la performance et de l'intérêt, manque d'entrain, interruptions volontaires, etc.

Toujours est-il que les sinistrés ont dû faire le deuil de leur ancienne maison, de leur terrain, de leurs objets personnels précieux et irremplaçables, certes, mais aussi de toutes ces 
petites choses qui font partie de la vie quotidienne (jardinière, légumier, soupière, nappe en dentelle, ustensiles, bicyclette, etc.). Chaque jour éveille le souvenir de l'ancienne demeure et rappelle les pertes: tantôt du bibelot rapporté d'un voyage, tantôt du cadeau d'un ami, tantôt des dessins des enfants, des albums de famille, etc. Bref, il faut loger dans un coin de sa mémoire les objets qui appartiennent aux expériences du passé tout en faisant le deuil de son ancien chez-soi. Et plus de deux ans après l'événement, la nouvelle demeure est considérée comme un lieu de transition, puisque l'on est incapable de recréer un milieu où l'on se sent en sécurité, un milieu qui nous ressemble.

\section{k) L'adaptation à une nouvelle vie}

Un changement de résidence commande l'obligation d'une réorganisation complète de soi-même et de sa famille. Dix-huit mois après la catastrophe, les individus se disent encore troublés, voire envahis par de profondes déceptions. L'anxiété, l'inquiétude et la peur viennent ébranler un quotidien empreint de regrets et de souvenirs. Ceux qui ont fait le choix de rester près de l'eau vivent régulièrement une forme d'insécurité. Cela étant, les stress consécutifs que les gens ressentent depuis plusieurs mois semblent affecter la capacité d'adaptation à un nouvel environnement. $\mathrm{Ce}$ changement suppose un ajustement sur le plan personnel et sur celui des relations avec les autres qui exige un dynamisme malheureusement enfoui sous une extrême fatigue.

\section{DISCUSSION ET CONCLUSION}

À la fin de cet exposé, une question surgit: "La liste d'événements qui est présentée ici est-elle susceptible de caractériser tout sinistre ou est-elle propre aux inondations qu'a connues le Saguenay en juillet 1996? » En d'autres termes : "Y a-t-il possibilité de généraliser davantage cette suite d'événements? ॥ II paraît probable que tous les individus qui perdent leur maison au moment d'un sinistre (inondations, tremblements de terre, incendies, etc.) sont susceptibles d'être confrontés aux difficultés et aux obstacles qui viennent d'être décrits. À des degrés divers ou avec certaines particularités peutêtre, mais il n'en demeure pas moins qu'une personne qui perd sa demeure doit nécessairement se reloger temporairement, 
déménager, effectuer une foule de démarches, tout en étant confrontée à une série de deuils. Bref, toutes les personnes qui, sans le vouloir, sont privées définitivement de leur domicile vivent une succession d'événements qui appellent l'adaptation à une nouvelle vie.

Au-delà du stress collectif que les inondations de juillet 1996 ont provoqué dans la communauté du Saguenay, ce désastre a entraîné les victimes dans un long parcours qui demeure laborieux plus de dix-huit mois après l'événement. En l'occurrence, écouter les sinistrés a permis de mieux comprendre les difficultés auxquelles ceux-ci ont dû faire face. II ressort que la suite d'événements stressants ont conduit les victimes sur un chemin plein de détours. En effet, l'itinéraire bien capricieux, subdivisé ici en onze épisodes, a soulevé des désordres physiques (insomnie, palpitations, tremblements, agitation, etc.) et psychologiques (anxiété, détresse, problèmes d'adaptation, etc.) ${ }^{3}$. De plus, ce découpage permet de mieux saisir ce que Murphy (1986) et Berren (1980) ont proposé en parlant d'événements traumatisants pour définir un désastre. Ce qu'il faut aussi comprendre, c'est que les inondations du Saguenay, et les principales pertes qui y sont associées, ont entraîné un état de désorganisation et de désorientation non seulement pendant les journées des 19, 20 et 21 juillet, mais pendant tous les nombreux mois qui ont suivi. En effet, durant cette période, les sinistrés ont dû faire face à une série d'événements stressants allant du relogement involontaire à l'adaptation qu'impose un nouvel environnement.

Les entrevues menées auprès d'une soixantaine de sinistrés permettent donc de montrer que le désastre qu'a connu la région du Saguenay se compose d'une suite d'événements qui se prolongent dans le temps. Donnée essentielle pour les intervenants sociaux qui sont appelés à travailler auprès des populations sinistrées pendant la crise et tout au long du processus de

Pour de plus amples informations sur les perturbations psychologiques que peut entraîner la perte de son domicile et de ses biens, voir les articles suivants : a) Maltais et collab., "Redéfinition de l'habitat et santé mentale des sinistrés suite à une inondation »; b) Lalande et collab., "Les sinistrés des inondations de 1996 au Saguenay: problèmes vécus et séquelles psychologiques », numéro spécial de la revue Santé mentale au Québec (volume XXV, numéro 1, printemps 2000). 
rétablissement. Ainsi, les intervenants doivent se montrer vigilants à toutes les étapes relevées, chacune d'elles appelant l'adaptation à des conditions inhabituelles. Et puisque les difficultés éprouvées ne sont pas seulement d'ordre émotif, mais aussi d'ordre matériel, financier et instrumental, le soutien apporté doit couvrir ces divers aspects. Les victimes ont besoin d'une aide concrète et de services particuliers susceptibles de répondre aux besoins spécifiques que chacun des événements engendre. Une aide qui non seulement est ponctuelle, mais qui s'étend sur une longue période, la fin de l'application des mesures d'urgence ne signifiant pas la fin des tourments. De plus, les nombreux deuils que doivent vivre les victimes (sur les plans récréatif, économique, social, etc.) exigent une grande vigilance de la part des intervenants pendant et après le sinistre. D'ailleurs, presque deux ans après la catastrophe, des victimes réclament encore de l'aide. En attendant, " ne me secouez pas, je suis plein de larmes », dira l'une d'entre elles en se rappelant les mots du poète.

\section{Suzie Robichaud}

Professeure-chercheure

Université du Québec à Chicoutimi

Danielle Maltais

Professeure-chercheure

Université du Québec à Chicoutimi

Gilles Lalande

Professeur-chercheur

Université du Québec à Chicoutimi

\section{Anne Simard}

Maîtrise en étude et intervention régionales

Université du Québec à Chicoutimi

Guy Moffat

Travailleur social (à la retraite)

Université du Québec à Chicoutimi 


\section{Références bibliographiques}

AMERICAN PSYCHIATRIC ASSOCIATION (1996). DSM-IV, Manuel diagnostique et statistique des troubles mentaux, traduction française, Paris, Masson.

APTEKAR, L. (1994). "The Psychology of Disaster Victims », dans L. Aptekar, Environmental Disasters in Global Perspectives, New York, G.K. Hall \& Co., p. 79-126.

BARTON, A.H. (1969). Communities in Disaster : A Sociological Analysis of Collective Stress Situations, New York, Anchor Books.

BELTER, R.W. et M.P. SHANNon (1993). « Impact of Natural Disasters on Children and Families », dans C.F. Saylor, Children and Disasters, Charleston, South Carolina, p. 85-103.

BerRen, M., A. Beigel et S. Ghertner (1980). "A Typology for the Classification of Disasters », Community Mental Health Journal, 17, p. 103-111.

BolIN, R. (1985). « Natural Disasters », dans Psychological Aspects of Disaster, R. Gist et S. Lubin (dir.), Colorado, p. 61-85.

BRESLAU, N. et G.C. Davis (1992). «Post-traumatic Stress Disorder in an Urban Population of Young Adults : Risk Factors for Chronicity », American Journal of Psychiatry, 48, p. 216-222.

Crabbs, M.A. et E. Hefrron (1981). «Loss Associated with Natural Disaster », The Personnel and Guidance Journal, vol. 59, $\mathrm{n}^{\circ} 6, \mathrm{p}$. 378-382.

DuFouR, J. (1997). " Les catastrophes naturelles exogènes et l'effet de serre. Quelques réflexions appliquées au déluge du Saguenay », dans J. Dufour (dir.), Les inondations de juillet 1996. Les premiers résultats du programme de recherche du GRIR à I'UQAC, p. 29-37.

FULLILOVE, M.T. (1996). «Psychiatric Implications of Displacement: Contributions from the Psychology of Place », American Journal of Psychiatry, vol. 153, no 12, p. 1516-1523.

Gleser, G.C., B.L. Green et C. Winget (1981). Prolonged Psychosocial Effects of a Disaster : A Study of Buffalo Creek, New York, New York Academic Press.

GoleC, J.A. (1983). A Contextual Approach to the Social Psychological Effects of a Disaster : A Study of Buffalo Creek, New York, New York Academic Press. 
GREEN. B.L. (1982). "Assessing Levels of Psychological Impairment Following Disaster Consideration of Actual and Methodological Dimensions », Journal of Nervous and Mental Disease, $\mathrm{n}^{\circ} 17, \mathrm{p}$. 544-552.

GREEN, B.L. et J.D. LINDY (1994). "Post-traumatic Stress Disorder in Victims of Disasters », Psychiatric Clinics of North America, vol. 17, $\mathrm{n}^{\circ} 2$, p. 301-309.

HARE, K. (1996). "Le réchauffement global: prudence et audace », Écodécision, hiver, $\mathrm{n}^{\circ} 19$, p. 29-32.

KINGston, W. et R. Rosser (1974). " Disaster : Effects on Mental and Physical State », Journal of Psychosomatic Research,18, p.437-456.

LebeL, C. (1997). Petites éternités où nous passons, Haïkus, Québec, Éditions Le Loup de Gouttière.

LeCHAT, M.F. (1979). " Disasters and Public Health », Bulletin of Public Health of World Organization, $\mathrm{n}^{\circ} 59$, p. 11-17.

Logue, J.N., M.E. Melick et H. HANSEN (1981). « Research Issues and Directions in the Epidemiology of Health Effects of Disasters », Epidemiologic Reviews, n 3, p. 140-162.

Maltais, D., S. Robichaud et A. Simard (1999). Le sinistre de juillet 1996 au Saguenay: conséquences sur la redéfinition de l'habitat, Ottawa, Société canadienne d'hypothèques et de logement.

Maltais, D., S. Robichaud, G. Lalande et A. Simard (1998). « De la stupeur à l'adaptation. Le long parcours des sinistrés », dans MarcUrbain Proulx (dir.), Une région dans la turbulence, Sainte-Foy, Presses de l'Université du Québec, p. 129-149.

MURPHY, S.A. (1986). «Perceptions of Stress, Coping and Recovery One and Three Years after a Natural Disaster », Issues in Mental Health Nursing, vol. 8, p. 67-77.

Ollendick, G. et M. Hoffman (1982). «Assessment of Psychological Reactions in Disaster Victims », Journal of Community Psychology, 4, p. 64-68.

PICKERING, K.T. et L.A. OWEN (1994). An Introduction to Global Environmental Issues, Londres, Routledge.

POWELL, B.J. et J. RAYNeR (1952). Progress Notes: Disaster Investigation July, 1, 1951, June 30, 1952, Englewood, Maryland, Army Chemical Center, Chemical Corps Medical Laboratories. 
RANGeLL, L. (1976). " Discussion of the Buffalo Creek Disaster: the Course of Psychic Trauma », American Journal of Psychiatry, 133, p. 313-316.

RAPHAEL, B. (1986). When Disaster Strikes: How Individuals and Communities Cope with Catastrophe, New York, Basic Books, Inc.

RAPHAEL, B. (1975). "Crisis and Loss: Counselling Following a Disaster », Mental Health in Australia, p. 118-122.

RowLES, G.D. (1983). "Place and Personal Identity in Old Age: Observations from Appalachia », Journal of Environmental Psychology, 3, p. 219-313.

SOLOMON, S.D., E.M. SMITH, T.R. Robins et R.L. PISCHBACK (1987). "Social Involvement as a Mediator of Disaster-induced Stress », Journal of Applied Social Psychology, vol. 17, p. 1092-1112.

SteinfieLD, E. (1982). «The Pace of Old Age : The Meaning of Housing for Old People », dans J.S. Duncan, Housing and Identity, Crosscultural Perspectives, New York, Holmes and Meier Publ., p. 198245.

Steinglass, D. et E. GerRity (1990). « Natural Disasters and Posttraumatic Stress Disorder : Short-term versus Long-term Recovery in Two Disaster-affected Communities », Journal of Applied Social Psychology, vol. 20, p. 1746-1765.

TURNER, B.A. (1976). "The Development of Disasters: A Sequence Model for the Analysis of the Origin of Disasters ", The Sociological Review, vol. 24, n 4 , p. 753-774.

TYHURST, J.S. (1951). "Individual Reactions to Community Disaster », American Journal of Psychiatry, vol. 107, p. 764-769.

URSANO, R.J. et J.E. MCCARROLL (1994). «Exposure to Traumatic Death : the Nature of the Stressor ", dans R.J. Ursano, B.G. McCaughey et C.S. Fullerton (dir.), Individual and Community Responses to Trauma and Disaster: The Structure of Human Chaos, Cambridge, Cambridge University Press, p. 46-71. 